\title{
Electron-Phonon Coupling in Silicene Nanoribbons
}

Jailson Gomes da Silva (PG), ${ }^{* 1}$ Luiz Antônio Ribeiro Junior (PQ), ${ }^{2}$ Geraldo Magela e Silva (PQ), ${ }^{2}$ Antônio Luciano de Almeida Fonseca (PQ), ${ }^{2}$ and Pedro H. de Oliveira Neto $(\mathrm{PG})^{2}$

${ }^{1}$ Federal Institute of Roraima, Roraima, Brazil, E-mail: jailson.silva@ifrr.edu.br

${ }^{2}$ Institute of Physics, University of Brasília, Brasília, Brazil

Abstract: We report the effects of electron lattice coupling on the charge density distribution of two dimensional silicene nanoribbons. By means of an extended tightbinding model with lattice relaxation, we provide theoretical explanation to the unexpected density of states, as well as other electronic properties observed in this kind of material. The methodology follows the Ehrenfest approximation. First, by using the self-consistent field scheme, the Hamiltonian's expectation value leads to an eigenvalue problem in terms of an initial set of coordinates. Under the stationary condition, the Ehrenfest scheme leads to an updated set of coordinates that depends on the eigenstates of the electronic problem. Then, one can use this new set of coordinates as input to the eigenvalue problem. The procedure is repeated until the achieving a given convergence criteria [1,2]. Spin-orbit coupling effects are introduced in order to treat the systems more accurately. The effect of the nanoribbon width is investigated and interesting patterns arise that are expected to greatly influence transport in these materials [3]. Importantly, an extensive analysis on the role played by electron-phonon lattice over the charge distribution is performed. Our results point towards the expected ranges that charge mobility might have in these materials. This contribution is expected to enlighten the behavior of charge distribution in silicone nanoribbon, which are systems of crucial technological appeal [4].

Key-words: silicone, electron-phonon coupling, charge distribution.

\section{References:}

[1] L. A. Ribeiro Junior and S. Stafström, Phys. Chem. Chem. Phys. (2015). DOI: 10.1039/C5CP06577A.

[2] P. H. de Oliveira Neto, J. F. Teixeira, W. F. da Cunha, R. Gargano, and G. M. e Silva. J. Phys. Chem. Lett., 2012, 3 (20), pp 3039-3042.

[3] Jia-An Yan, Ryan Stein, David M. Schaefer, Xiao-Qian Wang, and M. Y. Chou. Phys. Rev. B 88, 121403 (2013).

[4] Michelle J.S. Spencer, Tetsuya Morishita (Springer Series in Materials Science 235) (eds.) - “Silicene: Structure, Properties and Applications” - Springer International Publishing (2016). 